\title{
In situ Reduction of Silver Nanoparticles on Chitosan Hybrid Copper Phosphate Nanoflowers for Highly Efficient Plasmonic Solar-driven Interfacial Water Evaporation
}

\author{
Mei Zhang ${ }^{1}$, Wanghuai $\mathrm{Xu}^{1}$, Minfei Li ${ }^{1}$, Jiaqian $\mathrm{Li}^{1}$, Peng Wang ${ }^{2 *}$, Zuankai Wang ${ }^{{ }^{*}}$ \\ 1. Department of Mechanical Engineering, City University of Hong Kong, Hong Kong, 999077, China \\ 2. Department of Civil and Environmental Engineering, the Hong Kong Polytechnic University, Hong Kong, 999077, China
}

\begin{abstract}
The development of water purification device using solar energy has received tremendous attention. Despite extensive progress, traditional photothermal conversion usually has a high cost and high environmental impact. To overcome this problem, we develop a low cost, durable and environmentally friendly solar evaporator. This bi-layered evaporator is constructed with a thermal insulating polyvinylidene fluoride (PVDF) membrane as a bottom supporting layer and plasmonic silver nanoparticles decorated micro-sized hybrid flower $(\mathrm{Ag} / \mathrm{MF})$ as a top light-to-heat conversion layer. Compared with the sample with a flat silver film, the two-tier Ag/MF has a plasmonic enrichment property and high efficiency in converting the solar light to heat as each flower can generate a microscale hotspot by enriching the absorbed solar light. On the other hand, the PVDF membrane on the bottom with porous structure not only improves the mechanical stability of the entire structure, but also maintains a stable water supply from the bulk water to the evaporation interface by capillarity and minimizes the thermal conduction. The combination of excellent water evaporation ability, simple operation, and low cost of the production process imparts this type of plasmonic enhanced solar-driven interfacial water evaporator with promising prospects for potable water purification for point-of-use applications.
\end{abstract}

Keywords: bionic, plasmonic, water evaporation, synergistic effect, Ag NPs, hybrid flower

Copyright (C) The author(s) 2021.

\section{Introduction}

With the rapid population growth and ever-worsening environmental pollution, the shortage of fresh water resources is becoming a global problem, and severely restricts the further social and economic development ${ }^{[1,2]}$. Given the vast abundance of seawater, seawater desalination by various means, such as thermal desalination, reverse osmosis, electric dialysis, ion exchange method, has been widely employed to supplement much needed fresh water especially in severe water scare regions ${ }^{[3-7]}$. However, the seawater desalination processes all involves intensive energy consumption and emission of green-house gases, aggravate the ongoing energy crisis, and thus lead to adverse environmental impacts $^{[8,9]}$. Therefore, seawater desalination driven by a clean and renewable energy source is much needed ${ }^{[10]}$.

As one of the most renewable energies, solar energy has attracted a lot of attention ${ }^{[2,11]}$. Solar-driven water evaporation is emerging as a promising technology for seawater desalination and water purification, among others ${ }^{[12-14]}$. Though significant progress has been made to enhance solar water evaporation performance by designing and applying various solar absorbing materials ${ }^{[15-18]}$, the overall energy efficiency of the system is usually limited by the unnecessary energy consumption to heating up the entirety of bulk water ${ }^{[19]}$. Given the fact that water evaporation is an interfacial phenomenon where water molecules transform from liquid phase to vapor phase only at a thin interfacial boundary layer between air and water, developing a solar-driven evaporation system that harvests solar energy and heats water exclusively in the interfacial region is deemed energy-efficient ${ }^{[20]}$. An ideal solar-driven water evaporation system should engender an efficient upward liquid wicking and low thermal dissipation.

\footnotetext{
*Corresponding author: Zuankai Wang, Peng Wang

E-mail: zuanwang@cityu.edu.hk, peng1.wang@polyu.edu.hk
} 
In this work we report the development of a two-layered artificial skin for effective plasmonic solar-driven interfacial water evaporation. The structure of the top layer of the artificial skin resembles that of the leaves of Taro plant, which consists of Micro-sized Flower (MF) arrays covered by nanoscale hairs (Figs. 1a and 1b). Slightly different from the Taro plant leave, the micro-sized flowers are covered by plasmonic silver nanoparticles, referred to as microsized hybrid flower with Ag NPs (Ag/MF), which can enrich light absorption and serve as large-area Surface-Enhanced Raman Spectroscopy (SERS) "hot

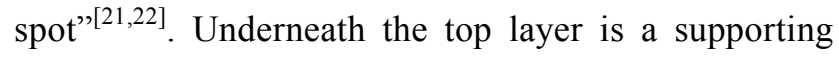
layer made of polyvinylidene fluoride (PVDF) membrane which is hydrophilic in wetting and has a low thermal conductivity. Thus, the SERS "hot spot" can effectively adsorb the light and convert them to heat, and meanwhile induces a continuous and spontaneous water pumping from the bulk water to the localized heating layer through inter-connected pores and minimizes heat transfer from the interface to the underneath bulk water. All together, this material exhibits an interfacial water evaporation performance and has a potential to be incorporated into solar distillation to produce potable water for off-grid communities.

\section{Materials and methods}

\subsection{Materials}

Copper (II) sulfate pentahydrate $\left(\mathrm{CuSO}_{4}\right)$, silver nitrate $\left(\mathrm{AgNO}_{3}\right)$, and chitosan were obtained from Sigma-Aldrich. Phosphate buffered saline (PBS, 1×, $\mathrm{pH}$ 7.4) was obtained from Invitrogen. All reagents were used as received without further purification. The aqueous solutions were prepared with deionized (DI) water.

\subsection{Nanoflower preparation}

Organic-inorganic hybrid nanoflower was prepared according to previously published protocols ${ }^{[23,24]}$. Briefly, $20 \mu \mathrm{L}$ of $120 \mathrm{mM} \mathrm{CuSO}_{4}$ solution were added to a solution of PBS containing different concentrations of prepared chitosan solution. The mixture was then left to incubate at room temperature for 3 days. After a reaction of 3 days, the precipitates (one-tier MF) were collected. To synthesize the two-tier Ag/MF, the resultant one-tier MF was washed using DI water for several times and dispersed in $1 \mathrm{mg} \cdot \mathrm{mL}^{-1} \mathrm{AgNO}_{3}$ solution to react for $5 \mathrm{~h}$. For Scanning Electron Microscopy (SEM) analysis, the suspension of nanoflowers was filtered and dried on a membrane (pore size $=0.1 \mu \mathrm{m}$ ) and sputter coated with gold. For Fourier-transform Infrared Spectroscopy (FTIR) analysis, the synthesized nanoflower precipitate was dried using lyophilization and collected for further experiment.

\subsection{Characterization}

The morphologies of the nanoflowers were characterized using SEM on a FEI/Quanta 450 microscope. The detection of the interfacial water evaporation was measured floating two-tier $\mathrm{Ag} / \mathrm{MF}$ in a quartz dish with DI water. The solar light was supplied by a Xenon lamp with a power density of $1.5 \mathrm{~kW} \cdot \mathrm{m}^{-2}$. Changes in the mass of water were measured with high-accuracy analytical balance. Infrared photographs were recorded with FLIR SC660. The thickness of PVDF membrane (hydrophobic and hydrophilic) is about $100 \mu \mathrm{m}$ and the pore size is $0.22 \mu \mathrm{m}$.

For the fabrication of two-tier $\mathrm{Ag} / \mathrm{MF}$ membrane, $10 \mathrm{mg}$ of two-tier $\mathrm{Ag} / \mathrm{MF}$ was dispersed in $10 \mathrm{~mL}$ of DI water by ultrasonication to make an aqueous dispersion. Then, the dispersion at the concentrations of $1 \mathrm{mg} \cdot \mathrm{mL}^{-1}$ was deposited onto a PVDF membrane filter $(50 \mathrm{~mm}$ in diameter, $0.02 \mathrm{~mm}$ pore size) to form an $\mathrm{Ag} / \mathrm{MF} / \mathrm{PVDF}$ membrane through vacuum filtration followed by drying under $\mathrm{N}_{2}$ flow. As a comparison, we also prepared one-tier MF/PVDF membrane by depositing MF $\left(1 \mathrm{mg} \cdot \mathrm{mL}^{-1}\right)$ onto a PVDF membrane. The one-tier Nano Silver (NS) membrane was prepared by depositing NS film onto a PVDF membrane using Physical Vapor Deposition (PVD) for $10 \mathrm{~s}$.

\subsection{Steam generation and solar desalination expe- riments}

The two-tier Ag/MF membrane was put on the surface of water in a beaker. Then the samples were irradiated by a solar simulator with a power density of $1.5 \mathrm{~kW} \cdot \mathrm{m}^{-2}$. The temperatures of the system were recorded by the infrared camera. The weight change was monitored by electronic analytical scale and then used to determine the evaporation rate and efficiency of solar steam generation. Here, we calculate the thermal 

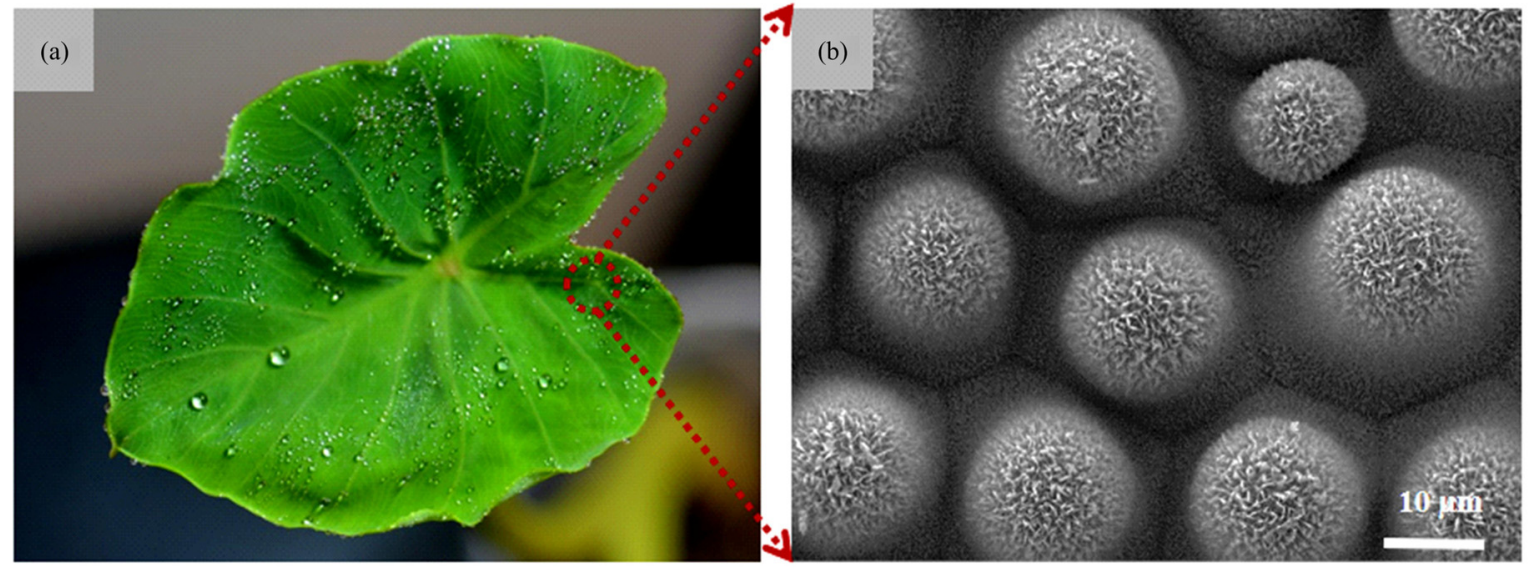

(c)
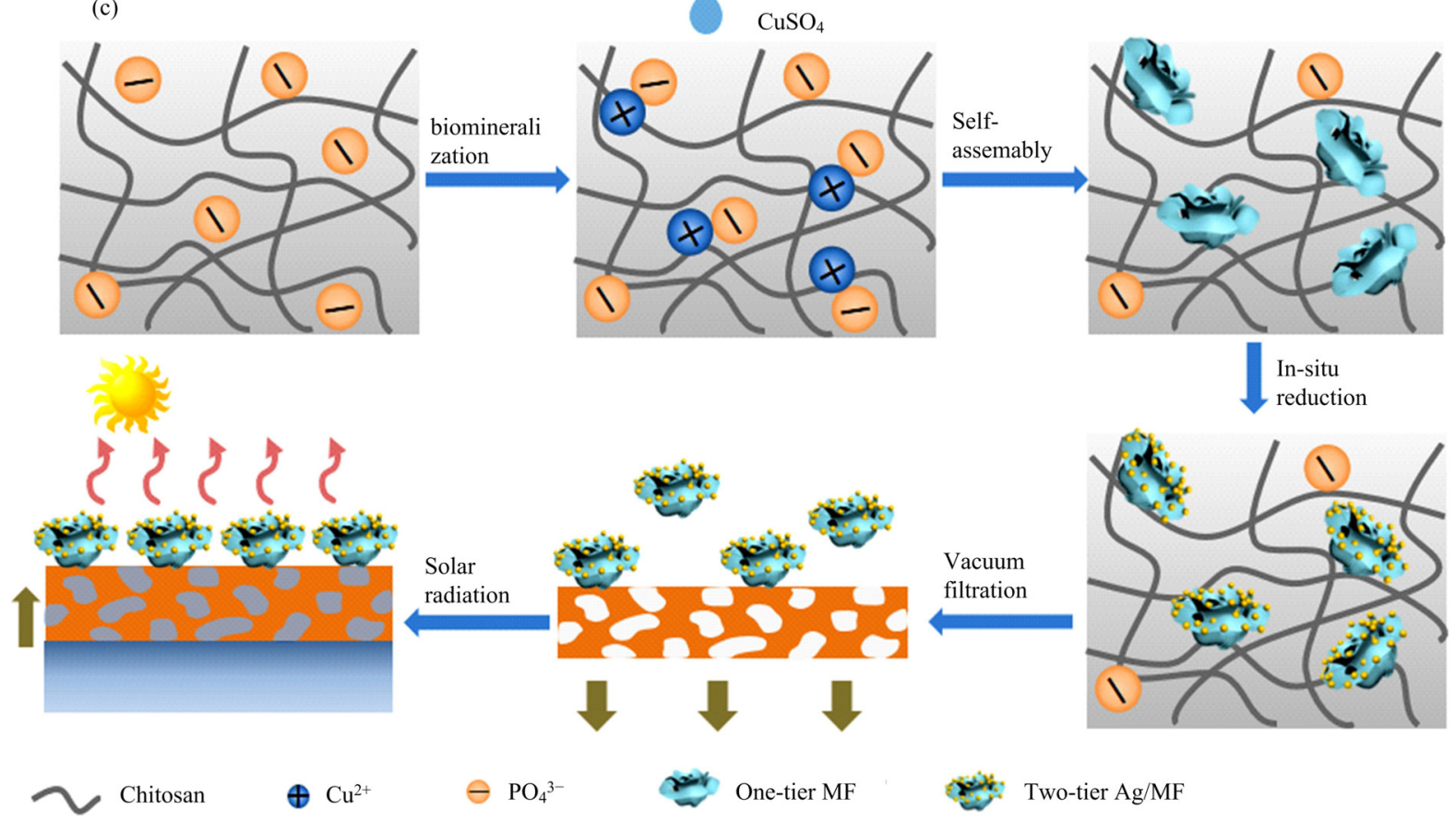

$$
\oplus \mathrm{Cu}^{2+} \quad \ominus \mathrm{PO}_{4}^{3-}
$$

One-tier MF

Two-tier Ag/MF

Fig. 1 Microstructure of natural taro leaf and bio-inspired nanostructures. (a) An optical image of a taro leaf; (b) a SEM image of taro leaf; (c) schematic illustration of preparation process of the bi-layered photothermal membrane with a porous two-tier Ag/MF on the top and hydrophilic PVDF membrane at the bottom. The two-tier Ag/MF was synthesized by a facile biomineralization and a redox reaction. Then the resultant $\mathrm{Ag} / \mathrm{MF}$ was deposited on the top of the PVDF membrane by vacuum filtration. The bi-layered membrane self-floated on the interface of air and water, captured solar energy, and converted it to the heat for water evaporation.

efficiency $\left(\eta_{\mathrm{th}}\right)$ to evaluate the performance of the membrane. $\eta_{\text {th }}$ was defined as follows:

$$
\eta_{\mathrm{th}}=m h_{\mathrm{LV}} / C_{\mathrm{opt}} q_{i}
$$

where $m$ is the mass flux, $h_{\mathrm{LV}}$ is the total enthalpy of the liquid-vapor change (sensible heat and phase-change enthalpy), $C_{\text {opt }}$ is the optical concentration (number of suns) and $q_{i}$ is the nominal direct solar irradiation $1 \mathrm{~kW} \cdot \mathrm{m}^{-2}$ ).

\section{Results and discussion}

We synthesized the $\mathrm{Ag} / \mathrm{MF}$ using a facile process, which is inspired by the kinetic control method which produces flower-like porous particles through the addition of copper ions into a PBS solution containing proteins ${ }^{[24]}$. The proteins in the solution act as the nucleation agent and capping agent during the preparation process. However, the use of proteins in the synthesis limits the 
further functionalization of the nanoflowers. In this work, we choose the utility of chitosan as a precursor to create hybrid organic-inorganic nanostructures with flower-like morphology. Fig. 1c schematically shows the synthesis of $\mathrm{Ag} / \mathrm{MF}$. Briefly, we first synthesized the one-tier MF by adding copper ion into PBS and chitosan solution. In this stage, the chitosan reacts with copper ions to form copper-chitosan complexes via coordination, which in turn serves as nucleation site in the self-assembly step to form particles branched structures, namely, MF. The as-prepared MF is then converted into two-tier $\mathrm{Ag} / \mathrm{MF}$ in the presence of silver nitrate via a redox reaction. Due to the reductive capability of chitosan, the two-tier $\mathrm{Ag} / \mathrm{MF}$ was synthetized by reduce silver nitrate to Ag NPs right in situ on the petals of the one-tier MF. After washing by de-ionized water for several times, the suspension containing the prepared $\mathrm{Ag} / \mathrm{MF}$ was filtered and dried on a PVDF membrane to finally give us the bi-layered taro-leaf-mimicking photo-thermal structure.

Fig. 2a shows the optical image of the as-developed artificial skin, in which the $\mathrm{Ag} / \mathrm{MF}$ is evenly dispersed on the surface of the commercial PVDF filter membrane with a stated pore size of $0.22 \mu \mathrm{m}$ using vacuum filtration. The skin is about $40 \mathrm{~mm}$ in diameter, blue in color, and integral in structure. Fig. $2 b$ shows the SEM images of individual two-tier Ag/MF on the PVDF membrane. As shown in the figure, the individual flower with a branched structure has a diameter of $40 \mu \mathrm{m}$, and the distance between the petals of the flower is about $0.78 \mu \mathrm{m}$ in average. The high-resolution SEM image of the magnified flower (Fig. 2c) displays that some nanoparticles with a diameter of $66.60 \mathrm{~nm}$ randomly distributed on the surface of the flower after an incubation of the hybrid flower with the silver nitrate solution. Compared with the two-tier Ag/MF, the surface of the untreated one-tier MF (Fig. 2d) is smooth. To further reveal the composition of the nanoparticles, we used Energy-dispersive X-ray spectroscopy (EDS) measurement to compare the change of the elemental and chemical composition of the hybrid flower before and after the treatment with the silver nitrate. The formation of copper phosphate and chitosan hybrid flower is further confirmed by the EDS measurement as shown in Fig. 2e. The elements of $\mathrm{Cu}(0.81 \mathrm{keV}$ and $0.93 \mathrm{keV}), \mathrm{P}(2.01 \mathrm{keV}$ and $2.14 \mathrm{keV})$ and $\mathrm{O}(0.53 \mathrm{keV})$ originate from copper phosphate and $\mathrm{C}(0.28 \mathrm{keV})$ and $\mathrm{O}(0.53 \mathrm{keV})$ comes from chitosan. Compared to the hybrid flower, there is an additional characteristic peak at $2.98 \mathrm{keV}$ and $3.16 \mathrm{keV}$ in the curve of two-tier $\mathrm{Ag} / \mathrm{MF}$, which belongs to silver element ${ }^{[25]}$.

We further measured the variation in the morphology of nanoflowers as a function of the concentration of chitosan. In the absence of chitosan, large porous structures (about $45 \mu \mathrm{m}$ in diameter) were formed, suggesting that the presence of chitosan is very important for the synthesis of flower-like particles. With an increase in concentration of chitosan $\left(0 \mathrm{mg} \cdot \mathrm{mL}^{-1}\right.$ to $0.5 \mathrm{mg} \cdot \mathrm{mL}^{-1}$ ), the density of nucleation sites increased correspondingly, and the distance between the petal decreased from $1.76 \mu \mathrm{m}$ to $0.48 \mu \mathrm{m}$. To verify the composition of the flower, FTIR was employed. As displayed in Fig. 2f, the peaks (in black) of at $628 \mathrm{~cm}^{-1}$ and $560 \mathrm{~cm}^{-1}$ represent the bending vibrations of $\mathrm{PO}^{3-}$ and $980 \mathrm{~cm}^{-1}$ represents the symmetric stretch of $\mathrm{PO}^{3-}$ groups $^{[26]}$. The composed and overlapped band in the $960 \mathrm{~cm}^{-1}-1200 \mathrm{~cm}^{-1}$ range is ascribed to the antisymmetric stretching vibration of $\mathrm{PO}^{3-}$. As shown in the curve of chitosan (in red), the characteristic peaks of chitosan appear at $3500 \mathrm{~cm}^{-1}(\mathrm{O}-\mathrm{H}$ and $\mathrm{N}-\mathrm{H}$ stretching vibration), $2875 \mathrm{~cm}^{-1}$ (C-H stretching vibration), $1650 \mathrm{~cm}^{-1}$ (N-H bending vibration), and $1070 \mathrm{~cm}^{-1}$ $(\mathrm{C}-\mathrm{O}-\mathrm{C} \text { stretching vibration })^{[27]}$. After careful comparison of the FTIR spectra of hybrid flower (in blue) with copper phosphate and chitosan, it is concluded that the hybrid flower has both characteristic peaks of copper phosphate $\left(628 \mathrm{~cm}^{-1}, 560 \mathrm{~cm}^{-1}\right.$ and $\left.980 \mathrm{~cm}^{-1}\right)$ and characteristic peaks of chitosan $\left(2875 \mathrm{~cm}^{-1}\right)$, indicating the successful hybridization of the two components.

To evaluate the solar-driven interfacial water evaporation performance of the two-tier $\mathrm{Ag} / \mathrm{MF}$, an Infrared (IR) spectroscopy camera was used to monitor the temperature change of water evaporation under simulated solar illumination under 1.5 sun illumination intensity with a power density of $1.5 \mathrm{~kW} \cdot \mathrm{m}^{-2}$. Upon the irradiation, the temperature of self-floating $\mathrm{Ag} / \mathrm{MF} / \mathrm{PVDF}$ rapidly increased from room temperature $\left(25{ }^{\circ} \mathrm{C}\right)$ to round $53.05{ }^{\circ} \mathrm{C}$ in Fig. $3 \mathrm{a}$, whereas the temperature of the PVDF membrane alone only reached to about $38.88^{\circ} \mathrm{C}$ in Fig. 3b. Compared with the PVDF, the 

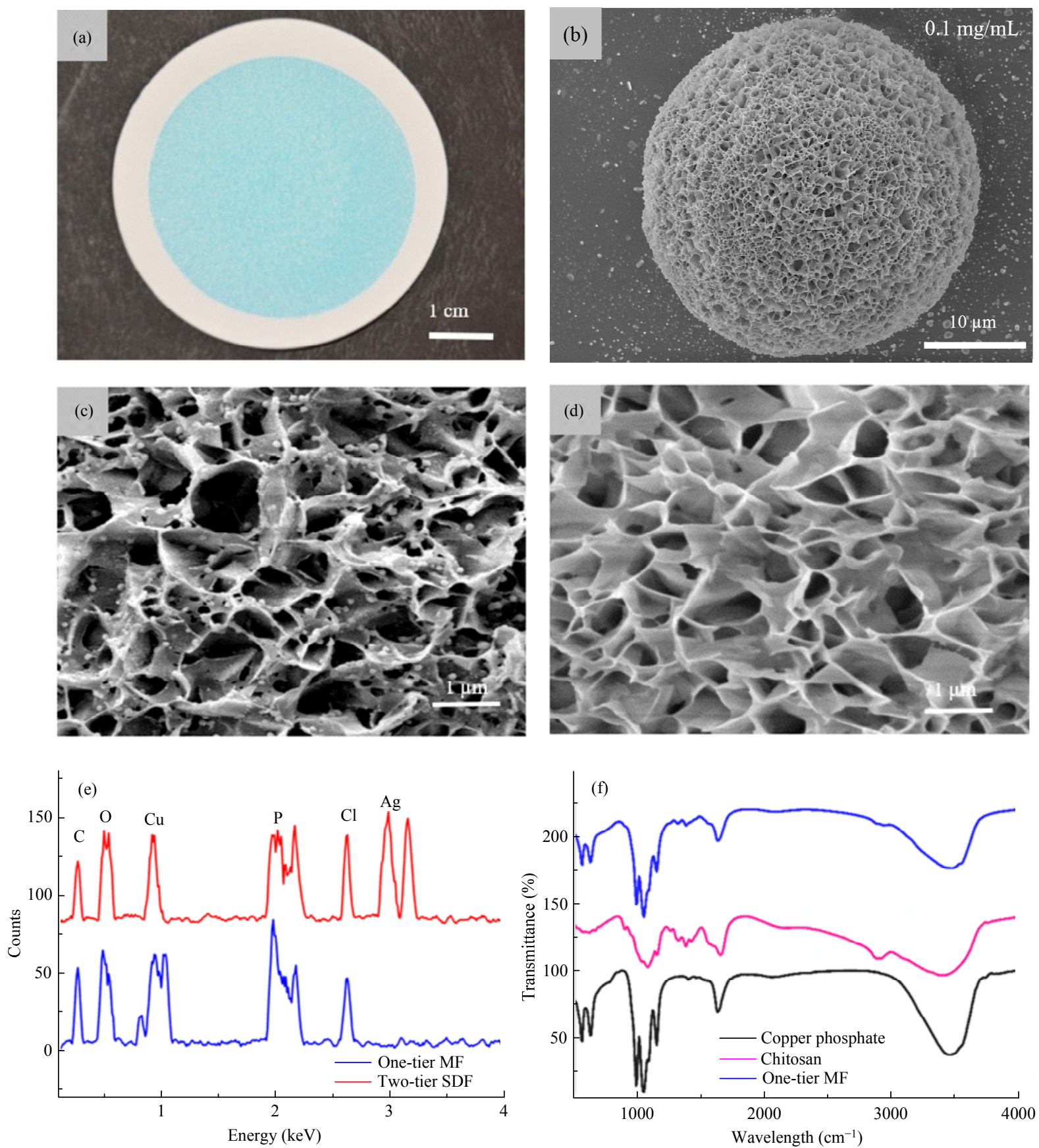

Fig. 2 The characterization of the two-tier Ag/MF. (a) An optical image of Ag/MF/PVDF membrane; (b) a SEM image of two-tier Ag/MF taro leaf-inspired nanostructures; a high-magnification SEM image of the MF (c) after and (d) before a reaction with silver nitrate; (e) EDS spectrums of one-tier MF and two-tier Ag/MF showing the presence of Ag NPs on the petals of the nanoflower; (f) FTIR spectra of copper phosphate nanoflower, pure chitosan and one-tier MF.

rapid increase of the two-tier Ag/MF's temperature is due to excellent light adsorbing capability of Ag NPs on the hierarchical flower-like architecture.

The two-tier Ag/MF layer with micro and nano structure acts as an effective photothermal layer as each flower on this layer can generate microscale thermal hotspot by enriching the solar light and subsequently converting the absorbed light to the heat. To verify our hypothesis, we monitored temperature of one-tier MF and one-tier NS on the PVDF membrane separately. As 
shown in Fig. 3b, the temperature of one-tier MF and one-tier NS floating on water surface increased from room temperature $\left(25{ }^{\circ} \mathrm{C}\right)$ to $47.04{ }^{\circ} \mathrm{C}$ and $49.13{ }^{\circ} \mathrm{C}$, respectively, lower than that of the two-tier $\mathrm{Ag} / \mathrm{MF}$ membrane (i.e., $53.05{ }^{\circ} \mathrm{C}$ ). This result confirms the synergistic effects of nano-sized $\mathrm{Ag}$ particles with plasmonic heating ability and hierarchical micro-sized porous structure for enhanced photothermal ability by enriching solar energy.

Interestingly, after an irradiation of $15 \mathrm{~min}$, the water temperature without the utility of the membrane reached about $41.23{ }^{\circ} \mathrm{C}$, which is $11.03{ }^{\circ} \mathrm{C}$ lower than that of the temperature of the PVDF membrane with two-tier Ag/MF. Such a difference in temperature between the PVDF membrane with two-tier Ag/MF and the bulk water might be due to the anisotropic thermal conductivity of PVDF membrane that helps heat confinement in the solar energy receiving layer by impeding the conductive heat transfer into the water. Note that the thermal conductivity of the PVDF membrane is measured
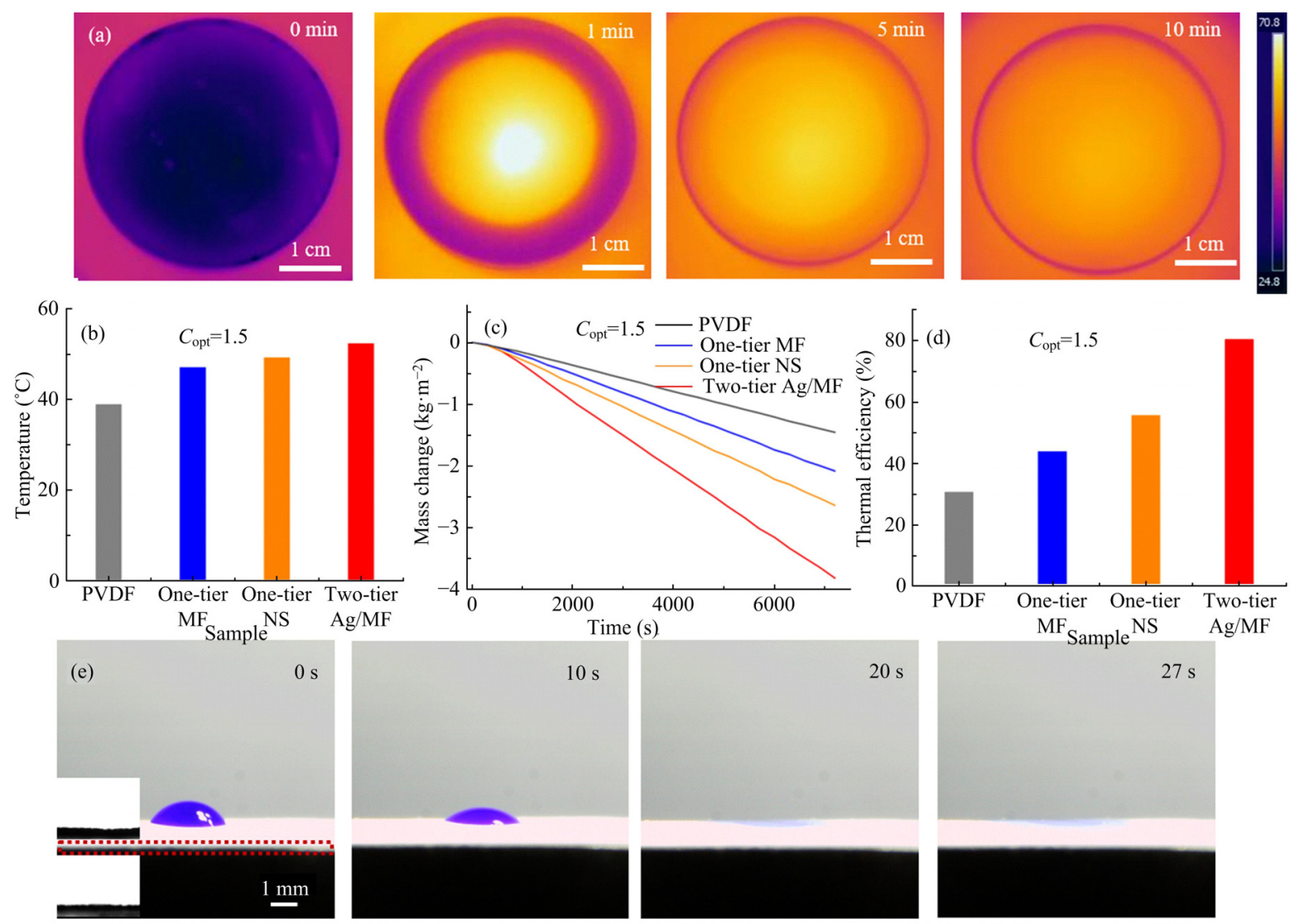

$C_{\mathrm{opt}}=1.5$
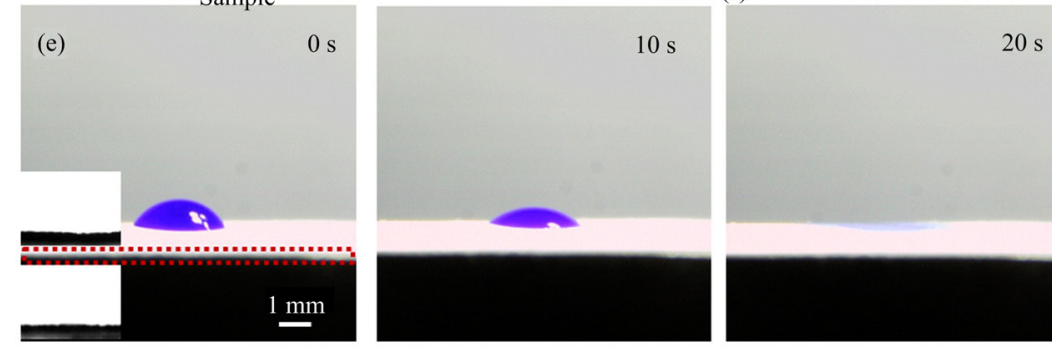

PVDF One-tier One-tier Two-tier

(f)
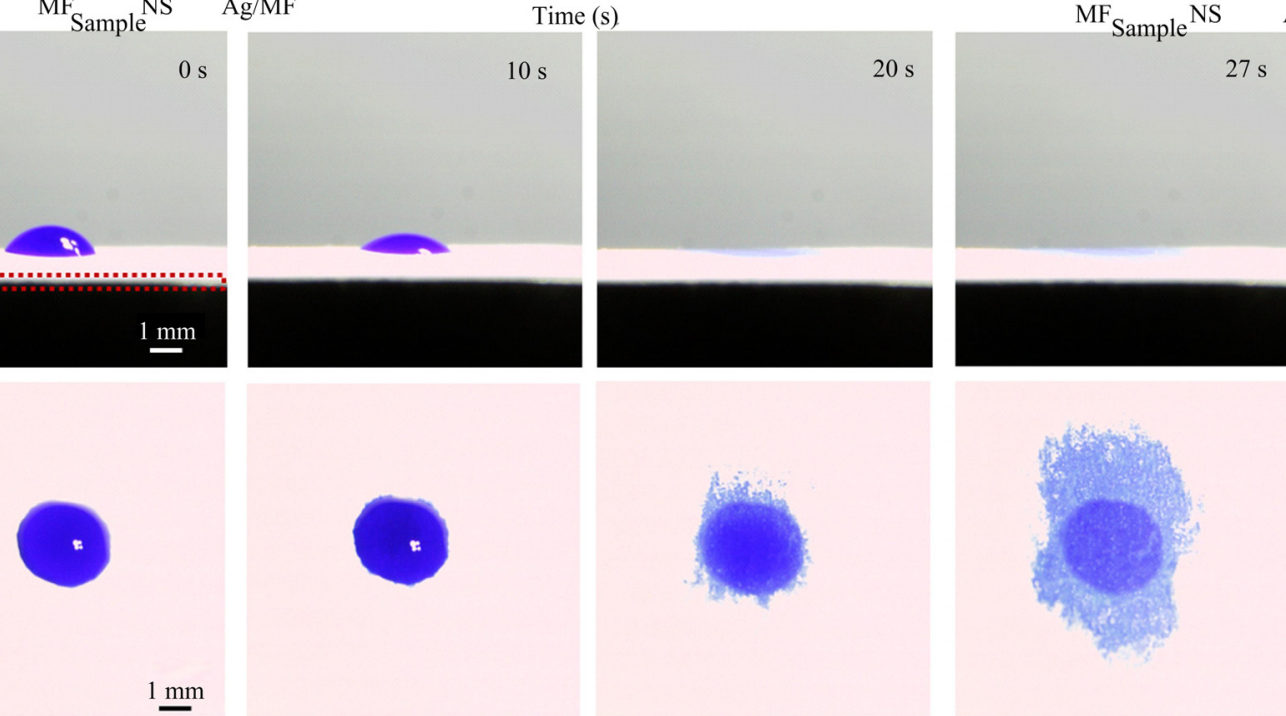

Fig. 3 Highly efficient photothermal steam generation using plasmonic two-tier Ag/MF. (a) Top view infrared images of hydrophilic PVDF membrane with two-tier $\mathrm{Ag} / \mathrm{MF}$ under a light illumination of $1.5 \mathrm{~kW} \cdot \mathrm{m}^{-2}$; comparison of the (b) temperature, (c) evaporation performance and (d) photothermal conversion efficiency of water covered with samples; (e) side and (f) top images of the water droplet penetration obtained by a high-speed camera at various durations. The water droplet can easily pass through the hydrophilic PVDF to two-tier Ag/MF side. The contact angle showed in the inset of (e) indicated the as-prepared membrane possessed a dual-hydrophilic surface with a contact angle of about $\sim 0^{\circ}$. 
to be $0.144 \mathrm{~W} \cdot \mathrm{m}^{-1} \cdot \mathrm{K}^{-1}$, which is lower than that of water $\left(0.600 \mathrm{~W} \cdot \mathrm{m}^{-1} \cdot \mathrm{K}^{-1}\right)$ yet higher than that of air $\left(0.024 \mathrm{~W} \cdot \mathrm{m}^{-1} \cdot \mathrm{K}^{-1} \text { at room temperature }\right)^{[28]}$.

To evaluate the steam-generation efficiency and desalination ability of two-tier $\mathrm{Ag} / \mathrm{MF}$, the weight loss of water due to the water evaporation was measured under simulated solar illumination. As shown in Fig. 3c, the weight loss of water was found to decrease linearly with time. After an irradiation of $2 \mathrm{~h}$, the weight loss of water from PVDF containing two-tier Ag/MF $\left(3.82 \mathrm{~kg} \cdot \mathrm{m}^{-2}\right)$ was $45 \%$ higher than one-tier NS $\left(2.64 \mathrm{~kg} \cdot \mathrm{m}^{-2}\right)$ and $83 \%$ higher than one-tier MF $\left(2.09 \mathrm{~kg} \cdot \mathrm{m}^{-2}\right)$. In comparison, the weight loss of water in the presence of an unmodified PVDF membrane was only $1.46 \mathrm{~kg} \cdot \mathrm{m}^{-2}$. The above results confirm the excellent evaporation performance of the Ag/MF/PVDF membrane.

The solar-to-water-evaporation efficiency $\left(\eta_{\text {th }}\right)$ was calculated to evaluate the performance of the membrane using Eq. (1). In Fig. 3d, the solar-to-water-evaporation efficiency of the two-tier $\mathrm{Ag} / \mathrm{MF}$ (80.33\%) is much higher than that of one-tier NS (55.56\%), one-tier MF (43.85\%) and bare PVDF membrane (30.64\%).

As shown in Fig. 3e, when a water droplet with a volume of $5 \mu \mathrm{L}$ was placed on the superhydrophilic Ag/MF/PVDF membrane, a water Contact Angle (CA) of $0^{\circ}$ in air was exhibited, with the droplet quickly permeating across from the PVDF side to the side with $\mathrm{Ag} / \mathrm{MF} / \mathrm{PVDF}$ within $30 \mathrm{~s}$ in side view. From a top view (Fig. 3f) by selected snapshots captured by the high-speed camera, the following mechanism of the droplet movement is suggested. At an early stage $(\leq 20 \mathrm{~s})$, the droplet first penetrates the PVDF membrane to the other side containing the hydrophilic two-tier $\mathrm{Ag} / \mathrm{MF}$ through capillary interaction. At a later stage ( $10 \mathrm{~s}$ to $20 \mathrm{~s}$ ), the penetrated droplet spreads in an irregular shape around the direction of the membrane. This above water transport behavior is helpful in pumping water from bulk water and delivering it to the water evaporation surface of two-tier Ag/MF.

To better understand the role of the PVDF substrate in water evaporation performance, we modified the wettability of the PVDF membrane and made corresponding comparison. After an irradiation of about $300 \mathrm{~s}$, the temperature of hydrophilic PVDF membrane $\left(52.30^{\circ} \mathrm{C}\right)$ was significantly higher than that of the hydrophobic
PVDF membrane $\left(48.71{ }^{\circ} \mathrm{C}\right)$ shown in Fig. 4a. The weight loss of water with hydrophilic PVDF membrane $\left(3.82 \mathrm{~kg} \cdot \mathrm{m}^{-2}\right)$ was also more than that with hydrophobic PVDF membrane $\left(3.59 \mathrm{~kg} \cdot \mathrm{m}^{-2}\right)$ after an irradiation for $2 \mathrm{~h}$ shown in Fig. 4b. As shown in Fig. 4c, the energy efficiency of the two-tier $\mathrm{Ag} / \mathrm{MF}$ on the hydrophilic PVDF membrane was $80.33 \%$ and that of the hydro-phobic PVDF membrane was $75.35 \%$. To explore the poor evaporation efficiency of hydrophobic PVDF membrane, a water droplet was added to the side with PVDF only of the Ag/MF/PVDF. The water droplet placed on the hydrophobic PVDF membrane had a CA of $125^{\circ}$ and kept its spherical shape within $30 \mathrm{~s}$ (Figs. 4d and 4e). Therefore, hydrophobic PVDF membrane with $\mathrm{Ag} / \mathrm{MF}$ restricted water evaporation by way of blocking-up fluid flow to the hot region. In comparison, the hydrophilic PVDF layer gave rise to a better and more stable water evaporation performance ${ }^{[29]}$. During the evaporation process, the light absorption by mesoporous Ag/MF on the hydrophilic PVDF led to a similar heat distribution across the wall, vapor can nucleate on both sides of the thermal cavity wall as shown in Fig. 4f. This was possibly enhanced by the higher internal light intensity. As the nanobubbles expanded, they coalesced to fill the inner thermal cavity and merged with the outer steam envelope.

\section{Conclusion}

With the rapid population growth and ever-worsening environmental pollution, the shortage of fresh water resources is becoming a global problem. Traditional water treatment methods usually have a high cost and high environmental impact. As a result, development of water purification device using clean energy has received tremendous attention. As one of the most renewable energies, solar energy has attracted a lot of attention in sea water desalination and water purification. Inspired by the structure of the taro leaf, we develop a facile and environmentally friendly synthesis of two-tier copper phosphate-chitosan hybrid nanoflower with evenly distributed Ag NPs. Due to the excellent plasmonic ability of the Ag NPs combined with enrichment of the hybrid flower, the resultant two-tier Ag/MF serves as large-area SERS "hotspot" and exhibits enhanced photothermal ability for water evaporation. The bottom 

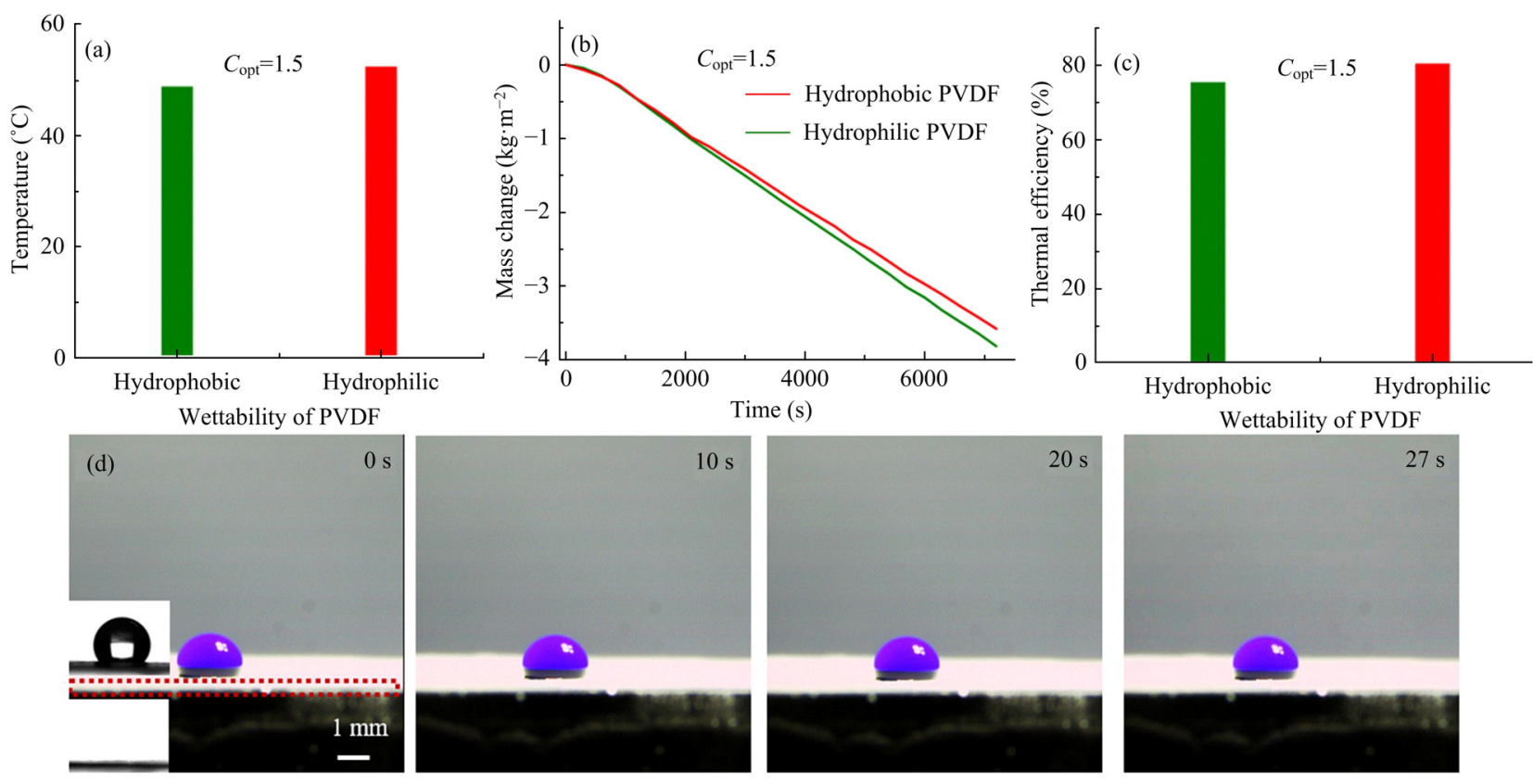

(e)
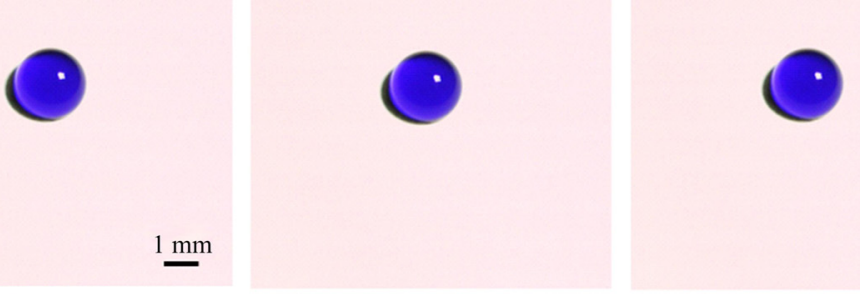

(f)
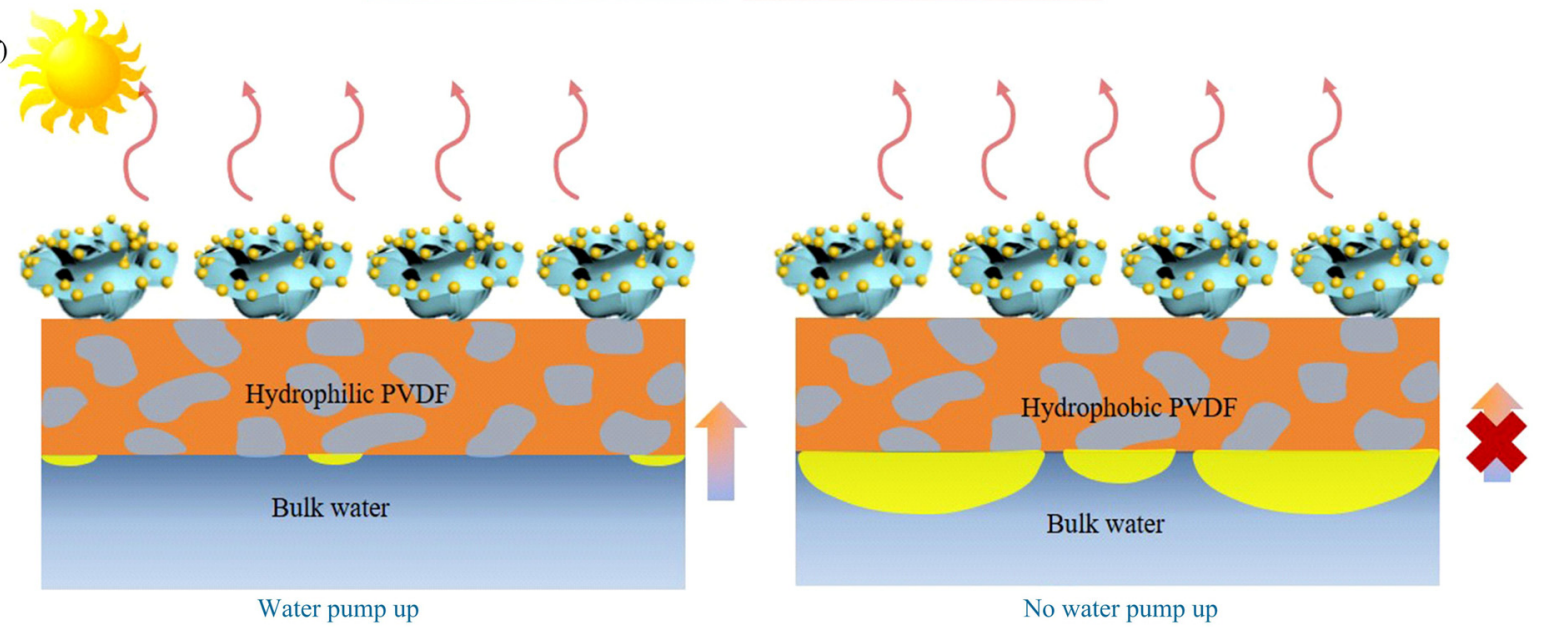

Fig. 4 Comparison of the efficient photothermal steam generation using two-tier Ag/MF on the PVDF with different wettability. (a) The temperature, (b) evaporation performance and (c) photothermal conversion efficiency of hydrophobic and hydrophilic PVDF membrane containing two-tier Ag/MF; (d) side and (e) top images of the water droplet are blocked by superhydrophobic PVDF obtained by a high-speed camera at various durations; (f) schematic of water evaporation process using PVDF membrane with different wettability. The water droplet keeps a spherical shape on hydrophobic PVDF. The contact angle shown in (d) indicated the as-prepared membrane possessed a hydrophilic surface with a contact angle of about $0^{\circ}$ and (c) a hydrophobic surface with a contact angle of about $125^{\circ}$. Both contact angle data were measured under dry conditions. 
PVDF membrane avoids the heat loss to the bulk water and maintains the transportation of water from the bulk water to the evaporation interface. In conclusion, this two-tier hybrid nanoflower with Ag NPs can effectively adsorb the light and convert them to heat and spontaneously pump water from the bulk water to the localized heating layer through inter-connected pores. This material exhibits an interfacial water evaporation performance and has a potential to be incorporated into solar distillation to produce potable water for off-grid communities.

\section{Acknowledgment}

We acknowledge the financial support from Shenzhen Science and Technology Innovation Council (No. JCYJ20170413141208098), Research Grants Council of Hong Kong (Nos. C1018-17G, 11275216 and 11218417), City University of Hong Kong (Nos. 9680212 and 9610375), Natural Science Foundation of China (No. 51475401).

Open Access This article is licensed under a Creative Commons Attribution 4.0 International License, which permits use, sharing, adaptation, distribution and reproduction in any medium or format, as long as you give appropriate credit to the original author(s) and the source, provide a link to the Creative Commons licence, and indicate if changes were made.

The images or other third party material in this article are included in the article's Creative Commons licence, unless indicated otherwise in a credit line to the material. If material is not included in the article's Creative Commons licence and your intended use is not permitted by statutory regulation or exceeds the permitted use, you will need to obtain permission directly from the copyright holder.

To view a copy of this licence, visit http://creativecommons.org/licenses/by/4.0/.

\section{References}

[1] Li X Q, Xu W C, Tang M Y, Zhou L, Zhu B, Zhu S N, Zhu J. Graphene oxide-based efficient and scalable solar desalination under one sun with a confined 2D water path. Proceedings of the National Academy of Sciences of the United States of America, 2016, 113, 13953-13958.

[2] Zhou L, Tan Y L, Wang J Y, Xu W C, Yuan Y, Cai W S, Zhu
S N, Zhu J. 3D self-assembly of aluminium nanoparticles for plasmon-enhanced solar desalination. Nature Photonics, 2016, 10, 393-398.

[3] Koschikowski J, Wieghaus M, Rommel M. Solar thermal-driven desalination plants based on membrane distillation. Desalination, 2003, 156, 295-304.

[4] Lee J B, Park K K, Eum H M, Lee C W. Desalination of a thermal power plant wastewater by membrane capacitive deionization. Desalination, 2006, 196, 125-134.

[5] Shannon M A, Bohn P W, Elimelech M, Georgiadis J G, Marinas B J, Mayes A M. Science and technology for water purification in the coming decades. Nature, 2008, 452, 301-310.

[6] Dabrowski A, Hubicki Z, Podkoscielny P, Robens E. Selective removal of the heavy metal ions from waters and industrial waste-waters by ion-exchange method. Chemosphere, 2004, 56, 91-106.

[7] Chen W, Chen S Y, Liang T F, Zhang Q, Fan Z L, Yin H, Huang K W, Zhang X X, Lai Z P, Sheng P. High-flux water desalination with interfacial salt sieving effect in nanoporous carbon composite membranes. Nature Nanotechnology, 2018, 13, 345-350.

[8] Liu Y M, Yu S T, Feng R, Bernard A, Liu Y, Zhang Y, Duan H Z, Shang W, Tao P, Song C Y, Deng T. A bioinspired, reusable, paper-based system for high-performance large-scale evaporation. Advanced Materials, 2015, 27, 2768-2774.

[9] Homaeigohar S, Elbahri M. Graphene membranes for water desalination. NPG Asia Materials, 2017, 9, e427.

[10] Liu Y, Lou J W, Ni M T, Song C Y, Wu J B, Dasgupta N P, Tao P, Shang W, Deng T. Bioinspired bifunctional membrane for efficient clean water generation. ACS Applied Materials \& Interfaces, 2016, 8, 772-779.

[11] Chen C J, Li Y J, Song J W, Yang Z, Kuang Y, Hitz E, Jia C, Gong A, Jiang F, Zhu J Y, Yang B, Xie J, Hu L B. Highly flexible and efficient solar steam generation device. $A d$ vanced Materials, 2017, 29, 1701756.

[12] Zhu L, Gao M, Peh C K N, Wang X, Ho G W. Self-contained Monolithic carbon sponges for solar-driven interfacial water evaporation distillation and electricity generation. Advanced Energy Materials, 2018, 8, 1702149.

[13] Ni G, Li G, Boriskina S V, Li H X, Yang W L, Zhang T J, Chen G. Steam generation under one sun enabled by a floating structure with thermal concentration. Nature Energy, 2016, 1, 16126.

[14] Jia C, Li Y J, Yang Z, Chen G, Yao Y G, Jiang F, Kuang Y D, Pastel G, Xie H, Yang B, Das S, Hu L B. Rich mesostructures 
derived from natural woods for solar steam generation. Joule, 2017, 1, 588-599.

[15] Zhu M W, Li Y J, Chen F J, Zhu X Y, Dai J Q, Li Y F, Yang Z, Yan X J, Song J W, Wang Y B, Hitz E, Luo W, Lu M H, Yang $\mathrm{B}, \mathrm{Hu}, \mathrm{L}$ B. Plasmonic wood for high-efficiency solar steam generation. Advanced Energy Materials, 2018, 8, 1701028.

[16] Zhou L, Tan Y L, Ji D X, Zhu B, Zhang P, Xu J, Gan Q Q, Yu Z F, Zhu J. Self-assembly of highly efficient, broadband plasmonic absorbers for solar steam generation. Science Advances, 2016, 2, e1501227.

[17] Liu Y M, Chen J W, Guo D W, Cao M Y, Jiang L. Floatable, self-cleaning, and carbon-black-based superhydrophobic gauze for the solar evaporation enhancement at the air-water interface. ACS Applied Materials \& Interfaces, 2015, 7, 13645-13652.

[18] Bae K, Kang G, Cho S K, Park W, Kim K, Padilla W J, Flexible thin-film black gold membranes with ultrabroadband plasmonic nanofocusing for efficient solar vapour generation. Nature Communications, 2015, 6, 10103.

[19] Shi L, Wang Y C, Zhang L B, Wang P. Rational design of a bi-layered reduced graphene oxide film on polystyrene foam for solar-driven interfacial water evaporation. Journal of Materials Chemistry A: Materials for Energy and Sustainability, 2017, 5, 16212-16219.

[20] Wang Y C, Zhang L B, Wang P. Self-floating carbon nanotube membrane on macroporous silica substrate for highly efficient solar-driven interfacial water evaporation. ACS Sustainable Chemistry \& Engineering, 2016, 4, 1223-1230.

[21] Huang J A, Zhang Y L, Zhao Y Q, Zhang X L, Sun M L, Zhang W J. Superhydrophobic SERS chip based on a Ag coated natural taro-leaf. Nanoscale, 2016, 8, 11487-11493.

[22] Zhang R, Xu B B, Liu X Q, Zhang Y L, Xu Y, Chen Q D,
Sun H B. Highly efficient SERS test strips. Chemical Communications, 2012, 48, 5913-5915.

[23] Zhang M, Peltier R, Zhang M M, Lu H J, Bian H D, Li Y Y, Xu Z T, Shen Y J, Sun H Y, Wang Z K. In situ reduction of silver nanoparticles on hybrid polydopamine-copper phosphate nanoflowers with enhanced antimicrobial activity. Journal of Materials Chemistry A: Materials for Energy and Sustainability, 2017, 5, 5311-5317.

[24] Ge J, Lei J, Zare R N. Protein-inorganic hybrid nanoflowers. Nature Nanotechnology, 2012, 7, 428-432.

[25] Venkatesham M, Ayodhya D, Madhusudhan A, Babu N V, Veerabhadram G. A novel green one-step synthesis of silver nanopar-ticles using chitosan: Catalytic activity and antimicrobial studies. Applied Nanoscience, 2014, 4, 113-119.

[26] Stoch A, Jastrzebski W, Brozek A, Stoch J, Szaraniec J, Trybalska B, Kmita G. FTIR absorption-reflection study of biomimetic growth of phosphates on titanium implants. Journal of Molecular Structure, 2000, 555, 375-382.

[27] Gregorio-Jauregui K M, Pineda M G, Rivera-Salinas J E, Hurtado G, Saade H, Martinez J L, Ilyina A, Lopez R G. One-step method for preparation of magnetic nanoparticles coated with chitosan. Journal of Nanomaterials, 2012, 8, 813958 .

[28] Liu K K, Jiang Q, Tadepallifit S, Raliya R, Biswas P, Naik R $\mathrm{R}$, Singamaneni S. Wood graphene oxide composite for highly efficient solar steam generation and desalination. ACS Applied Materials \& Interfaces, 2017, 9, 7675-7681.

[29] Yu S T, Zhang Y, Duan H Z, Liu Y M, Quan X J, Tao P, Shang W, Wu J B, Song C Y, Deng T. The impact of surface chemistry on the performance of localized solar-driven evaporation system. Scientific Reports, 2015, 5, 13600. 